Conference abstract POT11

\title{
P-gp Inhibitory Potential of Phospholipids
}

\author{
S. SIMON, R. SCHUBERT
}

Department of Pharmaceutical Technology and Biopharmacy, University of Freiburg, Germany

E-mail: silke.simon@pharmazie.uni-freiburg.de (S. Simon)

Sci Pharm. 2010; 78: 726

doi:10.3797/scipharm.cespt.8.POT11

Introduction:

Oral drug administration is mainly regulated by the step of intestinal absorption. For various substances uptake is strongly superimposed by an active efflux back into the lumen via ATP-driven pumps like the P-glycoprotein (P-gp), significantly decreasing their bioavailability.

P-gp inhibitory effects can be determined via the calcein accumulation assay (CAA) or transport studies in cellular systems. Hereby it was previously shown that particular phospholipids (PL) reduce the efflux of a common P-gp substrate [2].

The aim of this study is to identify further potent PL derivatives and their molecular mechanism of action and to investigate the influence of different experimental parameters.

Experimental Methods:

Cell culture: $\mathrm{CaCo} 2$ and MDCKII-mdr1 cells were routinely maintained in supplemented DMEM. Cells were grown for either 21 days in Transwell ${ }^{\circledR}$ plates in case of transport studies, or for 8 , resp. 4 days (MDCKII-mdr1) in 96-well plates in case of CAA.

Lipid formulations: Lipid derivatives were applied as liposomal formulations in HBSS.

Transport studies: CaCo2 cell layers were pre-incubated with lipid and digoxin $\left({ }^{3} \mathrm{H}\right.$-labeled) was added apically for absorptive, resp. basolaterally for secretory studies. Monolayer integrity was determined via transepithelial electrical resistance (TEER) measurements and the ratio of the apparent permeability coefficients $\left(\mathrm{P}_{\mathrm{app}}\right)$ of both directions displayed $\mathrm{P}$-gp effects.

$C A A$ : After pre-incubation with lipid or Verapamil as a positive control the intracellular accumulation of the fluorescent dye calcein indicated P-gp inhibition.

Results:

In the transport studies one achieved a concentration-dependent enhancement of netto drug absorption with C12-PG, C6-PS and various unsaturated symmetric and asymmetric PC lipids.CAA confirmed these findings in both cellines for C12-PG and other middle-chained saturated PC derivatives.

This work was financially supported by Phospholipid e.V., Heidelberg, Germany

[1] Zimmer C: Calcein-AM uptake assay for the screening of TPGS 1000 derivatives as potential Pglycoprotein inhibitors; Diploma Thesis, Saarbrücken, 2006.

[2] Alkatout A: Influence of phospholipids in nanoparticulate lipid carrier systems on the drug absorption across the duodenal epithelium determined via the CaCo2 cellular model; PhD Thesis, Freiburg, 2008.

[3] He W, Guo X, Zhang M. Transdermal permeation enhancement of N-trimethyl chitosan for testosterone. Int J Pharm. 2008; 356: 82-87. doi:10.1016/j.jpharm.2007.12.050 